\title{
COMPARAÇÃO ENTRE DIFERENTES EXERCÍCIOS RESISTIDOS PARA JOVENS NÃO TREINADAS
}

\section{Maristella Borges Silva}

Fisioterapeuta, Doutoranda em Engenharia Biomédica pela Universidade Federal de Uberlândia (UFU), Uberlândia (MG), Brasil.

\section{Fernando Max Lima}

Fisioterapeuta, Doutorando em Engenharia Biomédica Universidade Federal de Uberlândia (UFUBrasil; Docente Substituto da Universidade Federal do Triângulo Mineiro (UFTM), Brasil

Luciane Fernanda Rodrigues Martinho Fernandes

Fisioterapeuta, Doutora em Educação Física pela Universidade Estadual de Campinas (UNICAMP); Docente Associada da Universidade Federal do Triângulo Mineiro (UFTM), Brasil.

\section{Dernival Bertoncello}

Fisioterapeuta; Doutor em Ciências Fisiológicas; Docente Adjunto do Departamento de Fisioterapia Aplicada; Docente do Programa de Pós-graduação em Educação Física no Instituto de Ciências da Saúde, Universidade Federal do Triângulo Mineiro (UFTM), Brasil.

E-mail: bertoncello@fisioterapia.uftm.edu.br
RESUMO: O objetivo deste trabalho foi analisar e comparar os efeitos de 05 semanas de treinamento resistido sobre variáveis musculoesqueléticas, utilizando aparelhos de musculação ou massa corporal como resistência, em jovens não treinadas. Realizou-se estudo longitudinal, controlado, com 26 voluntárias divididas por sorteio simples em três grupos semelhantes: treinado com aparelho de musculação (GAM); treinado com resistência corporal (GRC); e sem treinamento - grupo controle (GC). Avaliações iniciais e reavaliações físicas após 05 semanas envolveram testes de flexibilidade, força e resistência muscular. Os grupos experimentais realizaram protocolos específicos de treinamento. De 15 variáveis analisadas, houve diferença significante em 09 favoráveis ao GRC e em 05 favoráveis aos GAM em relação ao GC. Houve diferença significante entre GRC e GAM somente para uma variável, favorável ao GAM. Constatou-se que 05 semanas de treinamento resistido foram suficientes para gerar alterações significantes sobre a flexibilidade, a força e a resistência muscular.

PALAVRAS-CHAVE: Força Muscular; Terapia por Exercício; Treinamento de Resistência.

\section{COMPARING DIFFERENT RESISTING EXERCISES FOR NON-TRAINED YOUNG PEOPLE}

\begin{abstract}
The effects of a five-week resisting training on muscleskeleton variables were compared. Bodybuilding or body mass apparatuses as resistance were employed by non-trained young people. The control longitudinal study was performed on 26 voluntary young people divided into three similar groups: trained with bodybuilding apparatus (GAM), trained with body resistance (GRC); non-trained (GC). After 5 weeks, physical initial and re-evaluations involved flexibility, force and muscular resistance tests. Experimental groups undertook specific training protocols. There was significant difference in 9 out of the 15 variables analyzed which were favorable to GRC and in 5 which were favorable to GAM, when compared to GC. There was a significant difference between GRC and GAM for a single variable, favorable to GAM. Results showed that five weeks of resisting training were sufficient to produce significant changes on flexibility, force and muscle resistance.
\end{abstract}

KEY WORDS: Therapy by Exercise; Resistance Training; Muscle Strength. 


\section{INTRODUÇão}

Os exercícios resistidos (ER) estão entre os mais difundidos, praticados e desenvolvidos mundialmente. Tal fato pode ser explicado pelos vários benefícios decorrentes de sua prática, que incluem modificações morfológicas, neuromusculares, fisiológicas, alterações sociais e comportamentais (DIAS et al., 2005). O treinamento com ER ficou popularmente conhecido pelo termo "musculação", sobretudo devido aos estabelecimentos comerciais (academias) onde sua prática mais se notabilizou. Entretanto, é preciso esclarecer que o ER não inclui apenas a realização de exercícios com pesos livres ou equipamentos de musculação, ele pode também ser realizado com resistências impostas através de dispositivos hidráulicos, de elásticos, molas, isometria e através da resistência do próprio corpo (ANUNCIAÇÃO; POLITO, 2011; KÜLKAMP et al., 2012; MENON; SANTOS, 2012; CESÁRIO et al., 2014; COELHO et al., 2014).

A mídia atual apresenta diferentes protocolos e técnicas de exercícios, bastante disseminados entre a população jovem, sejam para fins terapêuticos ou estéticos, muitos já com respaldo científico e boa aceitação pela comunidade acadêmica (CARVALHO; MOLINA; FONTANA, 2011; VILELLA; ZARCENO; ROSA, 2014). Também é encontrada a aplicação de exercícios resistidos para diferentes populações e com objetivos específicos (CAMARA et al., 2007; MUTTI et al., 2010; DUTRA et al., 2013).

Quando se fala em exercícios resistidos, há necessidade de se pensar nas diferentes formas de resistências aplicadas. A própria massa corporal pode ser utilizada como resistência, o que poderia ser útil para diferentes populações, incluindo especificidades clínicas (LIMA et al., 2012). Assim, a prática do exercício é possível em qualquer lugar, sem gastos, e pode ser adequada à disponibilidade de cada indivíduo ou terapêutica. Uma vez orientadas por profissionais habilitados sobre o protocolo de exercícios que serão realizados e seus cuidados, várias pessoas podem engajar-se em programas de treinamento resistido. No entanto, ainda são escassos, na literatura, relatos que demonstrem os efetivos benefícios corporais decorrentes de comparações dessas práticas, ou seja, entre diferentes formas de resistências para aplicação de exercícios, sejam a curto ou médio prazo.

Assim, objetivou-se analisar e comparar os efeitos de cinco semanas de treinamento resistido sobre o desempenho muscular de jovens não treinadas utilizando aparelhos de musculação ou massa corporal como resistência.

\section{METODOLOGIA}

\subsection{CARACTERIZAÇÃO DO ESTUDO}

Estudo experimental, controlado, longitudinal, aprovado pelo Comitê de Ética e Pesquisa da Universidade Federal do Triângulo Mineiro (UFTM), sob número 1483.

\subsection{PARTICIPANTES}

A amostragem, por conveniência, foi de 26 voluntárias não praticantes de atividades físicas regulares, com idades entre 18 e 25 anos, estudantes universitárias da UFTM. Foram considerados como critérios de exclusão: histórico de realização de musculação, nos últimos dois anos, por mais de seis meses consecutivos e falta em mais de 03 sessões consecutivas de treinamento. Um outro critério decisivo, para inclusão do participante na pesquisa, foi assinar o Termo de Consentimento Livre e Esclarecido.

Para início da pesquisa, todos os voluntários considerados aptos participaram de uma reunião explicativa sobre o andamento da pesquisa, bem como os procedimentos a serem adotados.

\subsection{PROCEDIMENTOS}

A cada participante foi apresentado um questionário referente aos dados gerais, como endereço completo, estado civil, período do curso realizado, tempo de prática de exercícios físicos regulares, tipo de exercícios realizados, atividades de vida diária, tipo de alimentação.

Em seguida, foi feita avaliação física para aferição da pressão arterial e frequências cardíaca e respiratória. Foram avaliadas as medidas antropométricas (estatura e massa corporal para a verificação de Índice de Massa 
Corporal), flexibilidade, força e resistência muscular.

Para quantificação da flexibilidade de cadeia muscular posterior foi realizado o teste de sentar e alcançar no Banco de Wells (RIBEIRO et al., 2010).

Foi avaliada a força de preensão palmar em dinamômetro hidráulico (Saehan ${ }^{\circledR}$, modelo SH 5001), sendo padronizada a posição da participante em ortostatismo, com ombro e antebraço em neutro e cotovelo flexionado a $90^{\circ}$. Foram realizadas 03 coletas, bilateralmente, com intervalo de 01 minuto entre cada medida, sendo considerada a média dessas (FERREIRA et al., 2011; VIVEIRO et al., 2014).

Para avaliação de força muscular, todas as participantes realizaram teste de 10 repetições máximas (10RM) com aparelhos de supino horizontal, extensores e flexores de joelho. Após cinco minutos de descanso do teste, foi solicitado que realizassem a maior quantidade de repetições possíveis até a exaustão com $80 \%$ das 10RM, a fim de se verificar a resistência muscular com uso dos aparelhos.
Ainda, todas as voluntárias realizaram testes motores para a quantidade máxima de repetições possíveis para flexão e extensão de braços no solo, agachamentos e abdominais durante 60 segundos e até exaustão. Os testes motores foram realizados somente com a resistência corporal, ou seja, sem incremento de carga extra (GUEDES, 2006).

Após 05 semanas, todas as participantes foram reavaliadas da mesma maneira da avaliação inicial.

\subsection{PROTOCOLOS DE TREINAMENTO}

Alongamentos globais eram realizados antes $\mathrm{e}$ após cada sessão de treinamento.

Os grupos experimentais, que praticaram exercícios com resistência corporal e exercícios com aparelho de musculação, GRC e GAM respectivamente, exercitaram-se durante 05 semanas, conforme descrito na Tabela 1, em dias não consecutivos. Cada sessão de treinamento teve uma hora de duração.

Tabela 1. Protocolos de Treinamento Utilizando Exercícios Resistidos

\begin{tabular}{|c|c|c|c|}
\hline Variáveis & GRC & GAM & GC \\
\hline Carga & Massa Corporal & $80 \%$ de $10 \mathrm{RM}$ & não se aplica \\
\hline Repetições baseadas em: & Repetições até exaustão & Repetições máximas com $80 \%$ de 10RM & \multirow{6}{*}{ não se aplica } \\
\hline $1^{\mathrm{a}}$ semana & $50 \%$ & $50 \%$ & \\
\hline $2^{\mathrm{a}}$ semana & \multirow{3}{*}{$75 \%$} & \multirow{3}{*}{$75 \%$} & \\
\hline $3^{\mathrm{a}}$ semana & & & \\
\hline $4^{\mathrm{a}}$ semana & & & \\
\hline $5^{a}$ semana & $100 \%$ & $100 \%$ & \\
\hline
\end{tabular}

$\overline{\mathrm{GRC}}=$ grupo treinado resistência corporal; GAM = grupo treinado aparelho de musculação; GC = grupo controle.

No intuito de padronizar a maior quantidade de variáveis durante o treinamento, as participantes foram orientadas a manter uma cadência de 03 segundos para cada ciclo completo em ambos os protocolos de treinamento, sendo 01 segundo para o movimento concêntrico e 02 segundos para o excêntrico.

\subsection{ANÁLISE DOS RESULTADOS}

Apósanálisededistribuiçãoetamanhodaamostra, optou-se pela utilização de estatística não paramétrica. Foram aplicados testes de Kruskal-Wallis previamente ao período de intervenção para qualificação da condição de igualdade entre os três grupos. Posteriormente, o mesmo teste foi aplicado para avaliar os valores finais e o delta de cada variável, sendo utilizado o teste post-boc de Dunn. Para análise intragrupos antes e após o período de intervenção foi realizado teste de Wilcoxon. A análise estatística foi realizada com auxílio do software BioEstat 5.3, sendo considerado nível de significância de 0,05 . O cálculo do poder da amostra através de força preensão palmar inicial do membro superior direito foi de 0,9009 (ANOVA One-way for several means). 


\section{RESULTADOS}

Todas as participantes apresentaram-se normotensas, normocárdicas e eupnéicas durante as avaliações (Pressão Arterial Sistólica de $120 \mathrm{mmHg}$, Pressão Arterial Diastólica de $80 \mathrm{mmHg}$, Frequência Cardíaca Média de 74 bpm, Frequência Respiratória de 17 irpm).

Quanto ao histórico de atividades físicas regulares, $58 \%$ já haviam realizado musculação com média de prática de 3,4 anos e 24,3\% possuíam histórico de prática esportiva ou outro tipo de treinamento físico com média de prática de 3,5 anos. A média de idade e índice de massa corporal de cada grupo estão na Tabela 2 .

Tabela 2. Idade e Indicadores Antropométricos da Amostra (Média \pm DP)

\begin{tabular}{llcc}
\hline & & Idade (anos) & IMC $\left(\mathbf{k g} / \mathbf{m}^{2}\right)$ \\
\hline GC & $(\mathbf{n}=9)$ & $22 \pm 2,1$ & $21,41 \pm 2,32$ \\
GAM & $(\mathbf{n}=\mathbf{8})$ & $23 \pm 2,7$ & $23,44 \pm 2,31$ \\
GRC & $(\mathbf{n}=9)$ & $21 \pm 1,4$ & $20,35 \pm 2,81$ \\
Total & $(\mathbf{n}=\mathbf{2 6})$ & $22 \pm 2,1$ & $22,24 \pm 2,37$ \\
\hline \multicolumn{4}{c}{ IMC = Índice de Massa Corporal; DP = desvio padrão. }
\end{tabular}

Em análise intra-grupos entre os testes pré e pós 05 semanas, não foram observadas diferenças significantes em relação ao GC. Já para o GRC, apresentaram aumentos significativos para dinamometria de preensão palmar (direita e esquerda), testes motores até exaustão (flexão e extensão de braços, agachamento e abdominais), testes motores em 1 minuto (flexão e extensão de braços), 10RM em movimentos padronizados (supino horizontal e extensão de joelhos) (Tabela 3).

O GAM apresentou aumentos significantes para os testes motores em 1 minuto (flexão e extensão de braços); testes motores até exaustão (agachamento); 10RM em movimentos padronizados (supino horizontal, extensão de joelhos e flexão de joelhos); repetições máximas com 80\% de 10RM (supino horizontal) (Tabela 3).

$\mathrm{Na}$ análise intergrupos, houve diferença significativa entre os grupos sob intervenção apenas no que se referia à quantidade de repetições executadas com 80\% das 10RM para flexão de joelhos, sendo maior para o GAM. Os valores nominais finais entre esses grupos apresentaram diferença significante somente para testes motores até a exaustão de agachamentos e abdominais.

Tabela 3. Valores de Mediana, Quartil Inferior e Quartil Superior por Grupos para os Momentos Pré-Treino, Pós-Treino eDiferença Entre os Anteriores, Respectivamente

(continua)

\begin{tabular}{|c|c|c|c|c|c|}
\hline Variáveis & GC & GRC & GAM & $p^{\prime}$ & p” \\
\hline \multirow[t]{3}{*}{ Din. D } & $28,3(26,0 ; 33,0)$ & $28,7(25,3 ; 29,0) \alpha$ & $26,3(20,7 ; 27,3)$ & 0.2634 & 0.50018 \\
\hline & $26,7(26,3 ; 33,3)$ & $32,3(32,0 ; 34,3)$ & $24,3(24,0 ; 30,7)$ & 0,0695 & 0.04311 \\
\hline & $0,7(0,3 ; 1,0) \mathrm{a}$ & $5,3(3,7 ; 8,3) b$ & $2,3(1,0 ; 3,3) a b$ & 0,0301 & 0.22491 \\
\hline \multirow[t]{3}{*}{ Din. E } & $25,7(23,3 ; 25,7)$ & $24,3(23,0 ; 24,3) \alpha$ & $23,3(22,0 ; 28,7)$ & 0.8852 & 0.27332 \\
\hline & $25,7(24,3 ; 28,0)$ & $27,3(26,7 ; 33,7)$ & $25,7(22,7 ; 29,7)$ & 0,3932 & 0.04311 \\
\hline & $0,6(0,0 ; 2,3) \mathfrak{a}$ & $5,0(4,3 ; 6,0) \mathrm{b}$ & $3,7(0,3 ; 4,0) \mathrm{ab}$ & 0,0392 & 0.13801 \\
\hline \multirow[t]{3}{*}{$\begin{array}{l}\text { Flexão/Extensão } \\
\text { braços }\end{array}$} & $20,0(13,0 ; 26,0)$ & $13,0(11,0 ; 13,0) \alpha$ & $17,0(11,0 ; 18,0)$ & 0.4966 & 0.50018 \\
\hline & $20,0(17,0 ; 22,0)$ & $28,0(20,0 ; 29,0)$ & $21,0(17,0 ; 25,0)$ & 0,2548 & 0.04311 \\
\hline & $-2,0(-5,0 ; 4,0) \mathrm{a}$ & $16,0(4,0 ; 17,0) \mathrm{b}$ & $3,0(3,0 ; 11,0) \mathrm{ab}$ & 0,0174 & 0.06789 \\
\hline $\begin{array}{l}\text { Agachamento } \\
\text { Exaustão }\end{array}$ & $53,0(50,0 ; 60,0)$ & $58,0(58,0 ; 70,0) \alpha$ & $45,0(40,0 ; 52,0) \alpha$ & 0.2497 & 0.68583 \\
\hline
\end{tabular}




\begin{tabular}{|c|c|c|c|c|c|}
\hline & $51,0(45,0 ; 57,0) \mathrm{a}$ & $174,0(142,0 ; 200,0) \mathrm{b}$ & $81,0(70,0 ; 134,0) a b$ & 0,0198 & 0.04311 \\
\hline & $-8,0(-9,0 ; 7,0) \mathrm{a}$ & $124,0(84,0 ; 130,0) \mathrm{b}$ & $30,0(18,0 ; 36,0) \mathrm{ab}$ & 0,0147 & 0.04311 \\
\hline \multirow[t]{3}{*}{$\begin{array}{l}\text { Abdominais } \\
\text { Exaustão }\end{array}$} & $30,0(30,0 ; 43,0)$ & $39,0(38,0 ; 41,0) \alpha$ & $30,0(27,0 ; 34,0)$ & 0.064 & 0.14412 \\
\hline & $34,0(29,0 ; 34,0) \mathrm{a}$ & $58,0(42,0 ; 60,0) \mathrm{b}$ & $35,0(33,0 ; 52,0) \mathrm{ab}$ & 0,0189 & 0.04311 \\
\hline & $-7,0(-9,0 ; 0,0) \mathrm{a}$ & $16,0(1,0 ; 22,0) \mathrm{b}$ & $14,0(5,0 ; 17,0) \mathrm{b}$ & 0,0418 & 0.07961 \\
\hline \multirow[t]{3}{*}{ SV 10RM } & $15,0(15,0 ; 23,0)$ & $16,0(14,0 ; 18,0) \alpha$ & $12,0(12,0 ; 15,0) \alpha$ & 0.417 & 0.85513 \\
\hline & $14,0(14,0 ; 15,0)$ & $22,0(16,0 ; 23,0)$ & $18,0(17,0 ; 20,0)$ & 0,2040 & 0.04311 \\
\hline & $0,0(-1,0 ; 1,0) \mathrm{a}$ & $5,0(5,0 ; 6,0) \mathrm{b}$ & $4,0(4,0 ; 6,0) \mathrm{b}$ & 0,0123 & 0.04311 \\
\hline \multirow[t]{3}{*}{ EJ 10RM } & $23,0(14,0 ; 30,0)$ & $17,0(12,0 ; 20,0) \alpha$ & $14,0(14,0 ; 20,0) \alpha$ & 0.4092 & 0.46520 \\
\hline & $22,0(17,0 ; 30,0)$ & $23,0(20,0 ; 25,0)$ & $22,0(22,0 ; 22,0)$ & 0,9823 & 0.04311 \\
\hline & $0,0(-1,0 ; 2,0) \mathrm{a}$ & $7,0(5,0 ; 9,0) \mathrm{b}$ & $8,0(2,0 ; 8,0) \mathrm{b}$ & 0,0390 & 0.04311 \\
\hline \multirow[t]{3}{*}{ FJ 10RM } & $10,5(7,0 ; 12,0)$ & $9,0(7,0 ; 10,0)$ & $8,0(7,0 ; 10,0) \alpha$ & 0.9328 & 0.28071 \\
\hline & $10,0(8,0 ; 12,0)$ & $12,0(10,0 ; 13,0)$ & $13,0(12,0 ; 15,0)$ & 0,5111 & 0.07961 \\
\hline & $1,0(-0,5 ; 1,5)$ & $3,0(2,0 ; 3,0)$ & $3,0(2,0 ; 6,0)$ & 0,1488 & 0.04311 \\
\hline
\end{tabular}

GC = grupo controle; GRC = grupo resistência corporal; GAM = grupo resistência de aparelhos; $\mathrm{p}^{\prime}=$ teste de Kruskal-Wallis para análise intergrupos; $\mathrm{p} "=$ teste de Wilcoxon para análise intragrupos; TSA = teste de Sentar e Alcançar; Din. $=$ dinamometria; $\mathrm{D}=$ direita; $\mathrm{E}=$ esquerda; Exaus = exaustão; $\mathrm{SV}=$ supino horizontal; $\mathrm{EJ}=$ extensão de joelhos; $\mathrm{FJ}=$ flexão de joelhos; $\mathrm{RM}=$ repetições máximas; $\mathrm{a} / \mathrm{b} / \mathrm{c}=$ diferenças intergrupos; $\alpha=$ diferença intragrupo entre os valores pré e pós treinamento. Os valores dispostos nas colunas do GC, do GRC e do GAM representam os valores iniciais, seguidos pelos valores finais e, por fim, a diferença entre os valores iniciais e finais para cada variável estudada. Os valores de $\alpha$ estão dispostos em coluna sendo o primeiro valor para o GC, o segundo para o GRC e o terceiro para o GAM.

Post Hoc Dunn. Nível de significância $<0,05$.

Conforme a Tabela 3, verificou-se ainda diferença significativa nos ganhos; também foi observada entre o GC e GRC para flexibilidade (TSA); dinamometria de preensão palmar (direita e esquerda); testes motores até exaustão (flexão e extensão de braços, agachamento e abdominais); testes motores em 01 minuto (flexão e extensão de braços); 10RM em movimentos padronizados (supino horizontal e extensão de joelhos).

Entre o GC e o GAM encontrou-se diferença significativa nos ganhos para um menor número de variáveis, nomeadamente: flexibilidade (TSA); testes motores até exaustão (abdominais); e 10RM em movimentos padronizados (supino horizontal e extensão de joelhos). Diferença nos valores nominais finais entre esses grupos não apresentaram diferenças significativas.

\section{DISCUSSÃO}

Alguns estudos discorrem sobre as influências de exercícios resistidos em mulheres idosas, homens jovens ou no tratamento de doenças (VALE et al., 2006; LUNZ et al., 2013; CESARIO et al., 2014; MYNARSKI et al., 2014). Poucos foram encontrados para o tipo de população aqui estudada.

Esta pesquisa constatou a importância da especificidade da tarefa, ou seja, os grupos sob intervenção, na análise intra-grupos, apresentaram melhora significante principalmente nos testes relacionados especificamente com o treino realizado. Porém, a melhora obtida em testes não específicos, diferentes ao treinamento realizado, foi significante 
somente em alguns casos, o que era esperado, uma vez que o aprendizado motor é fator preponderante nessa fase inicial do treinamento.

Somente o GRC apresentou diferença significante em relação à dinamometria de preensão palmar pré e pós-treinamento. Porém, essa diferença não se mostrou representativa ao final do período de intervenção na análise intergrupos. Outros trabalhos sugerem que a força de preensão palmar apresente alta correlação com a força de todo o membro superior ou mesmo com a força total do sujeito (BALOGUN; AKOMOLAFE; AMUSA, 1991). No entanto, protocolos de treinamento utilizando exercícios com sustentação do próprio corpo como resistência para membros superiores exigem maior atividade sinergista da musculatura do complexo antebraço, punho e mão, ao contrário de exercícios em máquinas específicas, comparando a flexão e extensão de braços e o supino horizontal, por exemplo. Isso favoreceria o ganho de força de preensão palmar e responderia os achados encontrados neste estudo, que correspondem a um maior aumento para o GRC.

$\mathrm{Na}$ análise intergrupos para os que tiveram intervenção, houve diferença significante apenas para os valores finais de quantidade de repetições para flexão de joelho com $80 \%$ das 10 RM, demonstrando maior resistência para a musculatura isquiotibial do GAM. Embora o GRC tenha realizado agachamentos capazes de ativar essa musculatura, apenas o GAM realizou exercícios específicos para essa musculatura, podendo ter influenciado este achado. Uma vez que outras diferenças não foram encontradas, supõe-se que os dois protocolos empregados possam ser eficazes de maneira similar para o treinamento de força e resistência muscular. Sabese, ainda, que o período de treinamento não pode ser indicativo de ganho de força pelo aumento de massa muscular. No entanto, os resultados encontrados já são promissores mesmo do ponto de vista da ativação da plasticidade neural.

Em outro trabalho (VALE et al., 2006), com maior período de intervenção, os autores observaram o efeito de 16 semanas de treinamento resistido para mulheres idosas sendo relatado aumento significante na força máxima e na flexibilidade. Ganho significante no componente flexibilidade encontrado no GRC pode estar relacionado ao fato de todas as participantes realizarem alongamentos globais previamente e ao final de cada sessão de treinamento impedindo, assim, a afirmação de qualquer efeito do treinamento resistido sobre a flexibilidade.

Em estudos realizados por outros autores (SALVADOR et al., 2005; DIAS et al., 2005), foi constatado que, para um protocolo de fortalecimento por oito semanas com iguais parâmetros para homens e mulheres, elas apresentaram um incremento relativamente superior na força muscular no teste de uma repetição máxima (RM) para os exercícios de supino reto, agachamento e rosca direta, demonstrando maior ganho de força muscular ao longo do tempo, apesar dos homens apresentarem força absoluta maior do que as mulheres em todos os exercícios analisados.

Outro trabalho encontrado, comparando diferentes protocolos de resistência, utilizou exercícios multiarticulares com resistência de aparelhos, dinamômetro isocinético e exercícios calistênicos e recreativos globais por 10 semanas, em idosos sedentários. Os resultados apresentados foram significativos a favor de exercícios em dinamômetro isocinético e com resistência de aparelhos para fortalecimento de quadríceps em detrimento de exercícios calistênicos (MALLIOU et al., 2003). Essas conclusões não corroboram com os achados deste trabalho, uma vez que ambos os grupos sob intervenção apresentaram melhoras em relação ao controle, porém, sem diferenças significantes entre si. No entanto, os próprios autores relatam a dificuldade em se comparar diferentes programas de fortalecimento, especificamente devido ao nível de força inicial dos indivíduos, ao volume, intensidade e frequência dos exercícios, aos equipamentos empregados e ao nível de atenção dos participantes durante a realização do programa.

Algumas limitações do estudo concentramse no fato de alguns dos testes empregados não serem padronizados, dificultando a comparação com outros estudos. Porém, com a utilização desses dados foi possível comparar a relação entre diferentes testes na avaliação da força e resistência muscular, em especial de membros inferiores. Em contrapartida, protocolos simples com testes de baixo custo e fácil aplicabilidade, 
como os utilizados com massa corporal como resistência, promoveram ganhos em uma maior quantidade de testes avaliados e podem ser empregados para favorecer o ganho de força e resistência muscular em pessoas não treinadas.

Outra limitação reside no fato de alguns estudos apontarem a variação no desempenho muscular em decorrência de flutuações hormonais durante o ciclo menstrual e utilização de contraceptivos orais (SARWARDE NICLOS; RUTHERFORD, 1996) ou de sintomas prémenstruais (GIACOMONI et al., 2000; CHAVES; SIMÃO; ARAÚJO, 2002). Entretanto, no presente estudo não foi analisado o uso de contraceptivos uma vez que outros autores não indiquem alteração significativa de fatores relacionados ao uso de contraceptivos ou da fase menstrual para ganho de força, o que seria importante para a amostra deste estudo por se tratar de estudantes jovens (LOUREIRO et al., 2011).

Chama-se a atenção para que estudos sejam desenvolvidos viabilizando a aplicação clínica de protocolos semelhantes no âmbito da promoção de saúde e nos diversos níveis de atenção à saúde e reabilitação, salvo suas especificidades. Outrossim, estudos que comparem diferentes tipos de resistência, durante poucas semanas de treinamento, são importantes para se verificar a eficácia de tais protocolos, uma vez que muitos deles são bastante disseminados pela mídia.

\section{CONCLUSÃO}

Apenas cinco semanas de treinamento resistido são capazes de gerar alterações significantes sobre a resistência muscular de jovens não treinadas. O protocolo de exercícios empregado utilizando massa corporal como resistência promoveu melhora em maior número de variáveis em relação ao grupo controle que o protocolo utilizando equipamentos de musculação.

\section{REFERÊNCIAS}

ANUNCIAÇÃO, P. G.; POLITO, M. D. Hipotensão pósexercício em indivíduos hipertensos: uma revisão. Arq Bras Cardiol., São Paulo, v. 96, n. 5, p. e100-e109, maio 2011.
BALOGUN, J. A.; AKOMOLAFE, C. T.; AMUSA, L. O. Grip strength: effects of testing posture and elbow position. Arch Phys Med Rehabil, Quebec, v. 72, p. 280-283, abr. 1991.

CAMARA, L. C.; SANTAREM, J. M.; WOLOSKER, N.; DIAS, R. M. R. Exercícios resistidos terapêuticos para indivíduos com doença arterial obstrutiva periférica: evidências para a prescrição. J. Vasc. Bras., Porto Alegre, v. 6, n. 3, p. 246-256, set. 2007.

CARVALHO, A. P. P. F.; MOLINA, G. E.; FONTANA, K. E. Suplementação com creatina associada ao treinamento resistido não altera as funções renal e hepática. Rev Bras Med Esporte, Niterói, v. 17, n. 4, p. 237-241, aug. 2011.

CESÁRIO, D. F.; MENDES, G. B. S.; UCHÔA, E. P. B. L.; VEIGA, P. H. A. Propcioceptive neuromuscular facilitation and strength training to gain muscle strength in elderly women. Rev Bras Geriat Gerontol., Rio de Janeiro, v. 17, n. 1, p. 67-77, jan. 2014.

CHAVES, C. P. G.; SIMÃO, R.; ARAÚJO, C. G. S. Ausência de variação da flexibilidade durante o ciclo menstrual em universitárias. Rev Bras Med Esporte, Niterói, v. 8, p. 212-218, nov. 2002.

COELHO, B. S.; SOUZA, L. K.; BORTOLUZZI, R.; RONCADA, C.; TIGGEMANN, C. L.; DIAS, C. P. Comparação $\mathrm{da}$ força e capacidade funcional entre idosos praticantes de musculação, hidroginástica e não praticantes de exercícios físicos. Rev Bras Geriatr Gerontol., Rio de Janeiro, v. 17, n. 3, p. 497-504, set. 2014.

DIAS, R. M. R.; CYRINO, E. S.; SALVADOR, E. P.; NAKAMURA, F. Y.; PINA, F. L. C.; OLIVEIRA, A. R. Impacto de oito semanas de treinamento com pesos sobre a força muscular de homens e mulheres. Rev Bras Med Esporte, Niterói, v. 11, n. 4, p. 224-228, ago. 2005.

DUTRA, M. T.; LIMA, R. M.; MOTA, M. R.; OLIVEIRA, P. F A.; VELOSO, J. H. C. L. Hipotensão pós-exercício resistido: uma revisão da literatura. Rev Educ Fis UEM, Maringá, v. 24, n. 1, p. 145-157, mar. 2013.

FERREIRA, A. C. C.; SHIMANO, A. C.; MAZZER, N.; BARBIERI, C. H.; ELUI, V. M. C.; FONSECA, M. C. R. Força 
de preensão palmar e pinças em indivíduos sadios entre 6 e 19 anos. Acta Ortop Bras., São Paulo, v. 19, n. 2, p. 92-97, abr. 2011.

GIACOMONI, M.; BERNARD, T.; GAVARRY, O.; ALTARE, S.; FALGAIRETTE, G. Influence of the menstrual cycle phase and menstrual symptoms on maximal anaerobic performance. Med Sci Sports Exerc., Indianapolis, v. 32, n. 2, p. 486-92, fev. 2000.

GUEDES, D. P.; GUEDES, J. E. R. P. Manual Prático para Avaliação em Educação Física. Barueri: Manole, 2006.

KÜLKAMP, W.; DIAS, J. A.; DOMENECH, S. C.; BORGES JUNIOR, N. G.; GEVAERD, M. S. Comparação do desempenho de praticantes de exercícios resistidos por diferentes métodos de ajuste pela massa corporal. Rev Bras Cineantropom Desempenho Hum., Florianópolis, v. 14, n. 3, p. 313-323, mai. 2012.

LIMA, F. M.; SILVA, M. B.; REZENDE, A. D.; FERNANDES, L. F. M. R.; MOTA, G. R.; Efeitos de exercícios com massa corporal como resistência em jovens não treinados. Conscient Saúde, São Paulo, v. 11, n. 3, p. 484-490, jun. 2012.

LOUREIRO, S.; DIAS, I; SALES, D.; ALESSI, I.; SIMÃO, R.; FERMINO, R. C. Efeito das diferentes fases do ciclo menstrual no desempenho da força muscular em 10 RM. Rev Bras Med Esporte, Niterói, v. 17, n. 1, p. 22-25, jan. 2011.

MALLIOU, P.; FATOUROS, I.; BENEKA, A.; GIOFTSIDOU, A.; ZISSI, V.; GODOLIAS, G.; FOTINAKIS, P. Different training programs for improving muscular performance in healthy inactive elderly. Isokinet and Exerc Sc., Amsterdam, v. 11, p. 189-195, 2003.

MENON, D.; SANTOS, J. S. Consumo de proteína por praticantes de musculação que objetivam hipertrofia muscular. Rev Bras Med Esporte, Niterói, v. 18, n. 1, p. 8-12, fev. 2012.

MUTTI, L. C.; SALLES, B. F.; LEMOS, A.; SIMÃO, R. Os benefícios dos exercícios resistidos na melhoria da capacidade funcional e saúde dos paraplégicos. Rev Bras Med Esporte, Niterói, v. 16, n. 6, p. 465-470, dec. 2010.
MYNARSKI, J.; SANTOS, L.; VERFFEL, A.; MELLO, D.; BERTICELL, M. W.; OLKOSKI, M. M. Efeitos de diferentes programas de exercícios físicos sobre a composição corporal e a autonomia funcional de idosas com risco de fratura. Rev educ fis UEM, Maringá, v. 25, n. 4, p. 609618, dez. 2014.

RIBEIRO, C. C. A.; ABAD, C. C. C.; BARROS, R. V.; BARROS NETO, T. L. Nível de flexibilidade obtida pelo teste de sentar e alcançar a partir de estudo realizado na Grande São Paulo. Rev Bras Cineantropom Desempenho Hum., Florianópolis, v. 12, n. 6, p. 415-421, jun. 2010.

SALVADOR, E. P.; DIAS, R. M. R.; CYRINO, E. S.; NAKAMURA, F. Y.; PINA, F. L. C.; OLIVEIRA, A. R. Comparação entre o desempenho motor de homens e mulheres em séries múltiplas de exercícios com peso. Rev Bras Med Esporte, Niterói, v. 11, n. 5, p. 257-261, jun. 2005.

SARWAR, R.; NICLOS, B. B.; RUTHERFORD, O. M. Changes in muscle strength, relaxation rate and fatigability during the human menstrual cycle. J Appl Physiol., Bethesda, v. 493, n. 1, p. 267-272, mai. 1996.

VALE, R. G. S.; BARRETO, A. C. G.; NOVAES, J. S.; DANTAS, E. H. M. Efeitos do treinamento resistido na força máxima, na flexibilidade e na autonomia funcional de mulheres idosas. Rev Bras Cineantropom Desempenho Hum., Florianópolis, v. 8, n. 4, p. 52-58, abr. 2006.

VILELLA, S. B.; ZARCENO, E. L.; ROSA, M. A. S. ¿Hay beneficios psicosociales por la práctica Pilates?: un análisis de la literatura científica. CPD, Murcia, v. 14, n. 3, p. 117-128, oct. 2014.

VIVEIRO, L. A. P.; ALMEIDA, A. S.; MEIRA, D. M.; LAVOURA, P. H.; CARMO, C. M.; SILVA, J. M.; TANAKA, C. Declínio de atividades instrumentais de vida diária associado à perda de força de preensão palmar em idosos internados em enfermaria pediátrica. Rev Bras Geriatr Gerontol, Rio de Janeiro, v. 17, n. 2, p. 235-242, mar. 2014.

Recebido em: 27 de setembro de 2015 Aceito em: 03 de novembro de 2015 